\title{
Estimation of intensive quantities in spatio-temporal systems from time-series
}

\author{
S. Ørstavik, R. Carretero-González ${ }^{1}$, J. Stark* \\ Centre for Nonlinear Dynamics and its Applications, University College London, Gower Street, London WC1E 6BT, UK \\ Received 22 December 1999; received in revised form 17 July 2000; accepted 7 August 2000 \\ Communicated by E. Kostelich
}

\begin{abstract}
We study multivariate time-series generated by coupled map lattices exhibiting spatio-temporal chaos and investigate to what extent we are able to estimate various intensive measures of the underlying system without explicit knowledge of the system dynamics. Using the rescaling and interleaving properties of the Lyapunov spectrum of systems in a spatio-temporally chaotic regime and paying careful attention to errors introduced by sub-system boundary effects, we develop algorithms that are capable of estimating the Lyapunov spectrum from time-series. We analyse the performance of these and find that the choice of basis used to fit the dynamics is crucial: when the local dynamics at a lattice site is well approximated by this basis we are able to accurately determine the full Lyapunov spectrum. However, as the local dynamics moves away from the space spanned by this basis, the performance of our algorithm deteriorates. () 2000 Elsevier Science B.V. All rights reserved.
\end{abstract}

Keywords: Spatio-temporal; Time-series; Lyapunov spectrum; Interleaving; Rescaling

\section{Introduction}

Spatio-temporal dynamical systems occur in many shapes and forms and are currently the subject of considerable interest. Typical everyday examples include the climate, ocean dynamics, biological systems such as the heart and the brain, road and telecommunication traffic, economic and social systems and many others. Such systems can be thought of as collections of many identical or similar sub-systems that interact (locally or globally) with each other. Many different approaches to the study of spatio-temporal systems exist. These can broadly be classified by whether or not one assumes that the dynamics of the system is

\footnotetext{
${ }^{*}$ Corresponding author.

E-mail address: j.stark@ucl.ac.uk (J. Stark).

${ }^{1}$ Present address: Department of Mathematics and Statistics, Simon Fraser University, Burnaby, BC, Canada V5A 1S6.
}

known. In the latter category fall time-series based techniques that attempt to categorise, predict or manipulate spatio-temporal systems purely on the basis of a time-series of repeated measurements of one or more observables (e.g. [1-7]).

In low-dimensional systems there is a well developed framework for the study of time-series generated by nonlinear dynamical systems (e.g. [8]). The first step is often the characterisation of the corresponding attractor using invariants such as the correlation dimension [9] and the Lyapunov spectrum (LS) [10]. These indicate the degree of complexity of the system, and can for instance be used to discriminate between time-series generated by different dynamical systems. They can thus often be used to eliminate potential mechanisms generating the data, and narrow the focus to models consistent with the measured quantities. 
In approaching multivariate time-series generated by spatio-temporal systems, it is a natural first step to attempt to compute analogous quantities. These can for instance indicate whether the system is in a spatio-temporally chaotic regime, or is better described by a model with only a few independent modes (and hence is exhibiting low-dimensional chaos). The computation of such invariants for spatio-temporal systems is, however, problematic. Such systems often have very high-dimensional attractors, with the dimension growing with the system's spatial extent. Since algorithms for the estimation of fractal dimensions and Lyapunov exponents require the available data to increase exponentially with the dimension of the attractor [11] it is not clear how they can be utilised in the context of spatially extended systems. One approach, suggested in [12,13] and further investigated in $[14,15]$, is to use the correlation dimension algorithm to calculate dimension densities. However, Torcini et al. [16] have questioned the ability of this method to distinguish between chaos and noise. Furthermore, even a small truncated lattice driven stochastically at its boundaries can model exponentially closely, the dynamics of a large (and even potentially infinite) lattice [17]. This suggests that one should not expect to be able to extract large fractal dimensions from localised time-series generated by such spatio-temporal systems.

An alternative approach, first suggested by Kaneko [18], is to estimate the Lyapunov spectrum. This relies on the rescaling property of the Lyapunov spectrum: it has been observed that for many systems exhibiting spatio-temporal chaos, the spectrum of a sub-system, when suitably rescaled, can give a good approximation of the spectrum of the whole spatio-temporal system [18-21]. Once the Lyapunov spectrum has been computed, other related quantities such as the Lyapunov dimension density and Kolmogorov-Sinai (KS) entropy density can also be determined. Unfortunately, there are a number of shortcomings to this method as it was described in [18]. Firstly, the method relies on a local approximation of the tangent map, which involves finding close neighbours in a reconstructed state space. Since even with a sub-system we have to work in embedding dimensions of least 20, it is not clear how such neighbours can be found with realistic amounts of data. Furthermore, Kaneko was not able to estimate the negative part of the Lyapunov spectrum accurately enough to be able to calculate the Lyapunov dimension density.

The aim of this paper is to address these shortcomings and suggest possible ways in which they may be overcome. Our starting point is the rescaling property described above, making use of the modified scaling introduced in [21] to significantly improve the accuracy of our estimates. In addition we use the interleaving property of successive sub-systems, first observed in [21], as a diagnostic tool. By interleaving we mean that the exponents of a system of size $N$ and $N+1$ are interlaced, that is the exponents of the smaller sub-system appear between the exponents of the larger ([21] and Fig. 1). This is reminiscent of the well known behaviour of the eigenvalues of symmetric matrices (e.g. [22]), where such interlacing can be rigorously proved (unlike in the case of exponents of spatio-temporal systems, where it remains an intriguing numerical observation).

Hitherto, rescaling and interleaving have been studied under the assumption that the dynamics of the system is known. We, therefore, carry out a systematic investigation of these properties when the system is estimated from time-series data. We find that interleaving is a robust property, and can be used as a diagnostic tool. We discuss how to carry out the rescaling (particularly in view of the more accurate rescaling introduced in [21]), and note that when the size of the whole system is not known a priori we cannot determine the actual spectrum of the whole system, but only its density.

It turns out that the boundary conditions at the edges of the sub-system can have a major influence on the accuracy of the estimated sub-system spectrum. In particular, in fitting elements of the Jacobian corresponding to sites at the boundary of a sub-system, the lack of data from outside the sub-system will bias our estimates. Essentially, the fitting algorithm compensates for this lack by introducing apparently random errors into the estimated Jacobian. We can overcome this by discarding the entries in the Jacobian corresponding to boundary sites. In effect, what we are doing is using 
data from a larger sub-system to obtain an unbiased estimate of the Jacobian for a smaller sub-system. Such truncation leads to a dramatic improvement in the accuracy of our estimates of the sub-system LS.

We also explore the effects of using higher order fits in estimating the Jacobian, and as in low-dimensional systems find that using these represents a worthwhile improvement over linear ones. Finally, we investigate a number of different choices of local dynamics in the spatio-temporal system, and conclude that the relationship between this and the space of functions used to fit the dynamics plays an important role in determining the accuracy of the final estimate. In particular, using restricted quadratic fits gives excellent results for a system where the local dynamics is given by the logistic map, but the estimates deteriorate as we skew this map, or replace it by an exponential one.

Our emphasis on interleaving, truncation and choice of basis extends the recent results of Bünner and Hegger [23], who present an algorithm to estimate the Lyapunov spectrum of spatio-temporal systems using a previously developed specific modelling approach [24]. Bünner and Hegger restrict themselves to the case of standard logistic map local dynamics, which as we shall see below is a relatively easy system for which to estimate the spectrum. They only consider local linear fits and do not investigate the effect of the fitting basis. Additionally, they use the whole of the estimated Jacobian in computing the sub-system Lyapunov spectrum, and as indicated above we have found that truncating this Jacobian leads to a major improvement in the accuracy of the resulting spectrum. We are currently developing a method that combines the best features of all of these approaches [25].

Our numerical work is carried out using coupled map lattices (CMLs), which are widely used as simple examples of spatio-temporal systems. Despite their simplicity, CMLs display a full range of complex spatio-temporal behaviour (e.g. [26]) and in particular as far we are aware can duplicate all of the behaviour (e.g. spatio-temporal chaos) of partial differential equations that is relevant to the present work. Furthermore, even though many models in physics are more naturally expressed using continuous space and time, when we sample observed data, or integrate a system numerically, we necessarily do so using discrete time and space, and hence effectively end up working with something resembling a CML.

Coupled map lattices can be thought of as a collection of local maps arranged on a spatial lattice and coupled together. The coupling is usually (but not always) restricted to some finite neighbourhood. More specifically, we focus on a one-dimensional CML with symmetric nearest neighbour coupling

$x_{i}^{n+1}=(1-\epsilon) f\left(x_{i}^{n}\right)+\frac{1}{2} \epsilon\left[f\left(x_{i-1}^{n}\right)+f\left(x_{i+1}^{n}\right)\right]$,

where $x_{i}^{n}(i=1, \ldots, N)$ is the state of the $i$ th site at discrete time $n, N$ the size of the system, $\epsilon$ the coupling strength, and we use periodic boundary conditions (i.e. $x_{i+N}^{n}=x_{i}^{n}$ ). We shall present results using three different choices of the local map $f$.

We should emphasise that throughout we work in parameter regions where the CML is believed to exhibit spatio-temporal chaos, or informally where it is fully turbulent. Situations where the system displays other kinds of dynamics, for instance low-dimensional chaos, travelling waves, various kinds of defects or similar are likely to need quite different approaches. In particular, one would not expect the Lyapunov spectrum or fractal dimensions to scale with system volume, and hence there would be no interleaving. This observation suggests that rescaling and interleaving can provide valuable diagnostic indications that the system is indeed spatio-temporally chaotic.

Although coupled map lattices are an ideal paradigm for the initial investigation of spatio-temporal phenomena, the framework adopted here is still some way from real data sets, say obtained from turbulent fluid flows or EEG recordings. We assume that we directly observe a one-dimensional dynamical variable at each site, with no noise, and restrict ourselves to nearest neighbour coupling and one spatial dimension. However, we feel that the work described here is a necessary first step towards tackling more realistic models, and it is preferable to develop and evaluate algorithms in the well controlled setting used here, rather than tackling all the complexities of real data. This sort of approach has worked well in time-series 
generated by low-dimensional systems [8]. Since rescaling and interleaving have also been observed for lattices with longer range coupling and two spatial dimensions [21], we are confident that the underlying ideas developed here will be relevant to more realistic systems.

\section{Lyapunov spectrum and related densities}

One invariant that one frequently wants to estimate from data generated by a spatio-temporal system is the spectrum of Lyapunov exponents [10]. This gives the average rate of exponential separation or convergence of two infinitesimally close initial conditions in phase space. In $N$-degrees of freedom system there are $N$ such exponents $\lambda_{i}, i=1, \ldots, N$, labelled such that $\lambda_{i} \geq \lambda_{i+1}$ which together form the LS. One or more positive exponents are taken as evidence that the dynamics of the system is chaotic. More specifically the Lyapunov exponents $\lambda_{i}$ are given by the logarithms of the eigenvalues of the matrix

$\Gamma=\lim _{n \rightarrow \infty}\left[P_{n}^{\mathrm{T}} P_{n}\right]^{1 / 2 n}$,

where $P_{n}=J_{n} J_{n-1} \cdots J_{2} J_{1}$ and $J_{k}$ is the Jacobian matrix along the trajectory at time $k$. The existence of the limit in Eq. (2) for almost every orbit (with respect to any given invariant measure) is guaranteed by the multiplicative ergodic theorem [27].

There is a close relationship between the LS and the fractal dimension of the system. The Lyapunov dimension $d_{\mathrm{L}}$ is given by [28]

$d_{\mathrm{L}}=j+\frac{\sum_{i \leq j} \lambda_{i}}{\left|\lambda_{j+1}\right|}$,

where $j$ is the greatest integer such that $\sum_{j} \lambda_{j}>0$. Although strictly not a dimension, $d_{\mathrm{L}}$ is usually a good numerical estimate of the fractal dimension. Another quantity that can be derived from the LS is the KS entropy. This quantifies the mean rate of information production in the system, or alternatively the mean rate of growth of uncertainty in a system subjected to small perturbations. An upper bound, and frequently a good estimate [10], for the KS entropy is given by the sum of the positive Lyapunov exponents, i.e.

$h=\sum_{i=1}^{N} \lambda_{i}^{+}$,

where $\lambda_{i}^{+}=\max \left\{\lambda_{i}, 0\right\}$.

In the case of spatially extended systems, e.g. in the case of a turbulent flow, it has been pointed out that sub-systems should be weakly correlated and thus the Lyapunov spectrum of the whole system should be approximately equal to the union of exponents from each non-interacting sub-system [19]. This implies that the LS should be intensive in that $\lambda_{i}=F(i / V)$ is a function of an intensive index $i / V$, where $V$ is the volume of the system. As a consequence, the Lyapunov dimension and KS entropy are extensive quantities that grow linearly with increasing system size $V$. Thus we can define the corresponding Lyapunov dimension density $\rho_{d}$ as

$\rho_{d}(V)=\lim _{V \rightarrow \infty} \frac{d_{\mathrm{L}}}{V}$,

and entropy density $\rho_{h}$ as

$\rho_{h}(V)=\lim _{V \rightarrow \infty} \frac{h}{V}$.

The densities defined in Eqs. (5) and (6) are intensive quantities that do not vary with system size. These quantities were first estimated from experimental data in [29].

Extensive studies have shown that for spatially extended systems, and in particular for coupled map lattices, the so-called sub-system LS converges rapidly towards the LS of the whole system for increasing sub-system size [18-21]. Instead of using all the $N$ variables of the system to build the Jacobian we only take a subset $N_{\mathrm{s}}$ of these variables (from $N_{\mathrm{s}}$ neighbouring sites) and build the Jacobian for this $N_{\mathrm{S}}$-dimensional sub-system without changing the underlying dynamics for the whole original system. As previously reported [21], when calculating the sub-system LS for increasing $N_{\mathrm{s}}$, one finds that Lyapunov exponents for two consecutive sizes are interleaved and that the sub-system LS converges to the LS for the whole system when rescaled suitably. More specifically, by interleaving we mean that the $i$ th Lyapunov exponent for the sub-system $N_{\mathrm{s}}$ lies 
between the $i$ th and $(i+1)$ th Lyapunov exponent of the sub-system $N_{\mathrm{s}}+1$. The interleaving property is discussed in some detail in [21]. The convergence to a limiting LS suggests that the Lyapunov dimension and KS entropy scale with sub-system size and this gives us the opportunity to estimate $\rho_{d}$ and $\rho_{h}$ as

$\rho_{d}(N)=\lim _{N_{\mathrm{s}} \rightarrow N} \frac{d_{\mathrm{L}}\left(N_{\mathrm{s}}\right)}{N_{\mathrm{s}}}$,
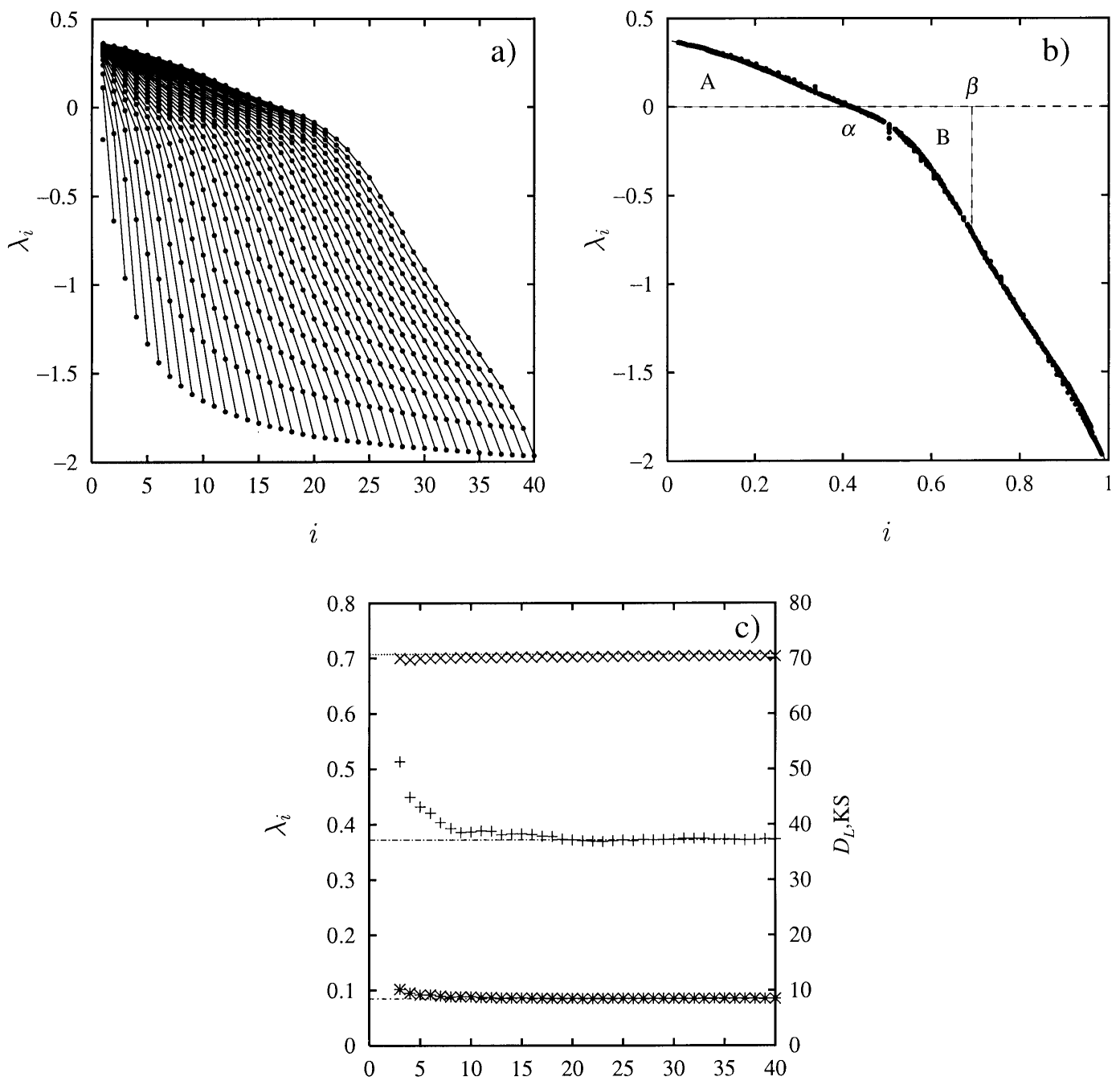

Fig. 1. Properties of the Lyapunov spectrum of a coupled map lattice as in Eq. (1) of size $N=100$ with $f(x)=4 x(1-x)$ and $\epsilon=0.45$. The spectrum has been calculated assuming full knowledge of the true dynamics. (a) Interleaving of sub-system LS for increasing sub-system sizes, $N_{\mathrm{s}}=1, \ldots, 40$. (b) Convergence to the limit curve using the new rescaling. The real LS is super-imposed as a continuous curve. The area $A$ equals the KS entropy density and the value of $\beta$ that makes area $A$ equal to area $B$ is an estimate of the Lyapunov dimension density. (c) Estimates of largest Lyapunov exponent $(+)$, Lyapunov dimension density $(\times)$ and KS entropy density $(*)$. 
the true dynamics. It is clear from this figure that in this case the Lyapunov exponents are perfectly interleaved. This suggests that there is convergence to a limit curve (the LS of the whole system) as seen in Fig. 1b. The usual method to rescale the sub-system LS is by plotting $\lambda_{i}$ against $r \times i$, where $r=1 / N_{\mathrm{s}}$ [18]. Here we have used a new rescaling introduced in [21] with

$r=\frac{N+1}{N\left(N_{\mathrm{s}}+1\right)}$.

This rescaling gives a better convergence to the real LS for small sub-system sizes. We see from Fig. 1b that the LS for the whole lattice is indeed well approximated. This convergence means that we are able to estimate the Lyapunov dimension and KS entropy. There are two ways of doing this from sub-system information. Firstly, one could use Eqs. (7) and (8) and plot $\rho_{d}\left(N_{\mathrm{s}}\right)$ and $\rho_{h}\left(N_{\mathrm{S}}\right)$ for increasing $N_{\mathrm{s}}$. In the thermodynamic limit (infinite number of lattice sites) these densities are intensive quantities (they do not depend on sub-system size). One can then estimate the Lyapunov dimension and KS entropy by multiplying by $N$. The second method, as suggested in [21], is to take the sub-system LS, rescale it, and then extrapolate a curve through it to obtain an approximation of the whole LS. A simple choice of extrapolation method is to use piece-wise linear fits. Thus, take the rescaled LS $\lambda_{i}\left(N_{\mathrm{S}}\right)$, and consider the polygon $\mathcal{P}$ through all the points $\left(i, \lambda_{i}\left(N_{\mathrm{s}}\right)\right)$. To estimate a Lyapunov exponent of the whole LS lying between $\lambda_{1}\left(N_{\mathrm{S}}\right)$ and $\lambda_{N_{\mathrm{s}}}\left(N_{\mathrm{s}}\right)$ one uses the fit given by the polygon $\mathcal{P}$. For Lyapunov exponents lying to the left (right) of the polygon use linear extrapolation from the first (last) two points of the rescaled LS. In this way the whole LS can be approximated and one can extract estimates of the largest Lyapunov exponent $\lambda_{1}$ and use Eqs. (5) and (6) to get the Lyapunov dimension $d_{\mathrm{L}}$ and the KS entropy $d_{h}$. This second method, together with the new rescaling has been found to give improved results [21]. In the example above, the estimates of Lyapunov dimension and KS entropy using the second method are seen (Fig. 1c) to converge rapidly to the exact values (70.7 and 8.46, respectively).
We now consider the case where only one or more time-series sampled from the system are available. Is it possible to get the same information as in the above example without knowing the dynamical equations governing the system? Ideally, we would like to be able to calculate Lyapunov exponents for sub-systems directly from time-series with some accuracy and one would hope that they converge to some limit curve for increasing sub-system size. It is clear that in this case we do not in general know the size of the full system, $N$. Hence we cannot use the rescaling in Eq. (9) to produce the limit curve that approximates the whole LS. However, since typically $N$ is large, we can assume $(N+1) / N \approx 1$ and therefore it seems plausible to use the approximate rescaling

$r=\frac{1}{N_{\mathrm{s}}+1}$,

when the system size $N$ is unknown. On the other hand, not knowing $N$ means that we are only able to estimate densities (and not the extensive quantities Lyapunov dimension and LS entropy for the whole system). We could choose to plot $\rho_{d}$ and $\rho_{h}$ for increasing $N_{\mathrm{s}}$ using Eqs. (7) and (8) and hope to observe convergence for $N_{\mathrm{s}}$ sufficiently large. Again, an alternative approach is to use the polygon method but modified as follows. The polygon can be considered as an approximation of the function $F(i / N)$, i.e. the whole LS. In the thermodynamic limit we can define $\rho_{h}$ as

$\rho_{h}=\lim _{N \rightarrow \infty} \int_{i=1}^{\alpha} F\left(\frac{i}{N}\right)$,

such that $F(\alpha)=0$. Note that the value of this integral corresponds to the area $A$ in Fig. 1b. Thus to get an approximation of $\rho_{h}, \rho_{h}\left(N_{\mathrm{s}}\right)$, from sub-system LS estimated from time-series, we calculate the area $A$ as given by the polygon. Similarly, we can find $\rho_{d}$ by solving for $\beta$,

$\int_{i=0}^{\beta} F\left(\frac{i}{N}\right)=0$.

This is equivalent to finding $\beta$ such that the areas $A$ and $B$ in Fig. 1b satisfy $A=B$. The corresponding $\beta$ is then an estimate of $\rho_{d}$. We will refer to this way of computing $\rho_{h}$ and $\rho_{d}$ from areas as the polygon 
method and call the estimation of $\rho_{d}$ and $\rho_{h}$ from Eqs. (7) and (8) as the direct method.

\section{Sub-system Lyapunov spectrum from time-series}

In the previous section the Lyapunov spectrum density and the related quantities $\rho_{d}$ and $\rho_{h}$ were calculated in the situation, where the equations governing the dynamics of the system are fully known. In the rest of this paper we examine to what extent we are able to reproduce these results when the only information available to us is some time-series sampled from different spatial locations. We have already discussed how to utilise sub-system Lyapunov spectra to estimate the various densities. We, therefore, now focus on how to find the sub-system Lyapunov exponents themselves from a time-series.

Usually the LS is calculated from an observed time-series using the method of time delays [10]. Given a scalar time-series $u_{i}$ one aims to reconstruct the dynamics of the original system by forming time delay vectors $U_{i}=\left\{u_{i}, u_{i-1}, \ldots, u_{i-d+1}\right\}$, where $d$ is the embedding dimension. If we use a sufficiently large $d$ then $\left\{U_{i}\right\}$ is a reconstruction of the original dynamics. We can then use this to estimate the Jacobians and subsequently the LS. However, using the method of delays to obtain good estimates of the LS is known to be difficult in practice, even for low-dimensional systems. Spatially extended systems, which we are interested in here, are typically high-dimensional and thus the extraction of the LS is even more difficult. Takens theorem [30] requires us to work in dimensions larger than $2 M$, where $M$ is the dimension of the state space of the system. Since the method involves finding neighbouring points in high dimensions, this means that we require an enormous amount of data to have any hope of decently reconstructing the dynamics.

Here we consider the case where $N_{\mathrm{s}}$ time-series are observed at $N_{\mathrm{s}}$ different neighbouring spatial locations simultaneously. Space delay vectors are built up using data from neighbouring sites as entries. If we have measurements $x_{i}^{m}$ taken at sites $i=1, \ldots, N_{\mathrm{s}}$ and time $m$, we build the vectors $V_{i}^{m}=\left\{x_{i}^{m}, x_{i+1}^{m}, \ldots, x_{i+d-1}^{m}\right\}$. Intuitively one would expect that the added information included by utilising several sites at once would increase our ability to estimate the LS.

Consider space delay vectors of size $d$. We can treat these as sub-systems of size $N_{\mathrm{s}}=d$ as above and aim to estimate the densities from the corresponding reconstruction. It should be emphasised that when using time-series we assume that the size of the original system is unknown. Therefore, only densities can be approximated since they do not depend on the system size. Their extensive counterparts $d_{\mathrm{L}}$ and $h$ obviously cannot be estimated without knowing $N$.

The Lyapunov exponents can be extracted from time-series by applying the algorithm introduced in [10]. The method consists of estimating the Jacobians along a trajectory and reorthogonalizing using QR decomposition. From the data we construct $d$-dimensional space delay vectors $v^{n}=$ $\left(x_{1}^{m}, x_{2}^{m}, \ldots, x_{d}^{m}\right)$. Note that this is a pure spatial delay reconstruction; we are not including any temporal information. Let us denote by $v_{j}^{m}, j=1, \ldots, k, k$ is the nearest neighbours of $v^{m}$, using the $l_{2}$-norm as distance metric. We can then define $k$ distance vectors $z_{j}^{m}=v^{m}-v_{j}^{m}$. The neighbourhood matrix $B_{m}$ at time $m$ is then defined by

$B_{m}=\left(z_{1}^{m}, z_{2}^{m}, \ldots, z_{k}^{m}\right)^{\mathrm{T}}$,

where $(\cdot)^{\mathrm{T}}$ denotes transpose. The image of this matrix is simply given by

$B_{m+1}=\left(z_{1}^{m+1}, z_{2}^{m+1}, \ldots, z_{k}^{m+1}\right)^{\mathrm{T}}$,

and the Jacobian $J(m)$ at time $m$ is then estimated by $J_{m}=B_{m+1}^{+} B_{m}$,

where $(\cdot)^{+}$denotes the pseudo-inverse. This is equivalent to finding $J_{m}$ by using least squares.

Now, in order to find the Lyapunov exponents recall that we look at the growth of $d$-volumes under the action of $J_{m}\left(v^{0}\right)$. Let $Q_{0}$ be a unit $d$-cube (matrix) defined by orthogonal vectors $\left\{q_{1}, \ldots, q_{d}\right\}$. The Lyapunov exponents are then given by the logarithms of the eigenvalues of $J_{m}\left(v^{0}\right) Q_{0}$ as $m \rightarrow \infty$. A serious problem when using this limit is that this matrix is 
ill-conditioned. To avoid this we use the approach suggested in [10] and reorthogonalize at each step. This can be achieved using Gram-Schmidt, Householder or Givens based techniques. Here, we use an efficient Householder method, where only the terms required for the computation of the Lyapunov exponents are calculated [31].

In the case discussed above, the Jacobian can be estimated by a local linear fit. It is also possible to include higher order terms in the neighbourhood matrix, e.g. by considering second-order terms. The hope is that by including these, the estimate of the Jacobian will improve, hence giving us a better approximation of the Lyapunov exponents. As a special case let us choose the space delay vectors as

$v^{m}=\left(x_{1}, x_{2}, \ldots, x_{d},\left(x_{1}\right)^{2},\left(x_{2}\right)^{2}, \ldots,\left(x_{d}\right)^{2}\right)$,

where all entries are evaluated at time $m$. This choice is a reasonable compromise between a linear fit and a full quadratic fit, which would include all the cross-terms of the form $x_{i} x_{j}$. The difficulty with the latter is that it substantially increases the number of parameters that we need to fit, and hence the number of nearby data points that we require. Since in our particular system the diffusive coupling acts linearly, it is reasonable to expect that cross-terms will be relatively insignificant, and a basis of the form (13) gives a reasonable approximation to a full quadratic fit.

The neighbours and neighbourhood matrices are now found using these vectors and the Jacobian is given by the upper-left $d \times d$ sub-matrix of $T_{m}$. The problem with including higher order terms is that the number of neighbours needed to fit the Jacobian grows rapidly for increasing embedding dimension $d$ and the data requirements soon get too large. In fact, the minimal number of neighbours required to fit the Jacobian (including cross-terms) with an embedding dimension $d$ is $\prod_{k=1}^{d}[(d+k) / k]-1$.

A further significant improvement in the calculation of the sub-system LS can be made by consideration of the boundary conditions of the sub-system, and the effect of this has on the Jacobian. When a sub-system Jacobian is estimated from observed data, the input at the edges of the sub-system acts as noise and interferes with the accurate determination of the Jacobian.
In particular, consider a site at the boundary of the sub-system. Its dynamics depends on a neighbouring site which is not explicitly contained in the measurement window. As a consequence, when we estimate the dynamics at this site as a function of the observed data, the fitting algorithm will attempt to compensate for the missing data by spuriously adjusting the estimated values at other sites. Thus for instance, the fitting procedure for the first row of the Jacobian will offset the lack of information from the neighbouring site on the left (which is outside of the measurement window) by assigning biased values to the sites within the window. Such a 'noisy' Jacobian leads to a loss of accuracy in estimating the sub-system LS.

We can overcome this by discarding the outer layer of the Jacobian (i.e. the first and last row and column) once it has been fitted. This ensures that the entries in the Jacobian that is used to compute the LS are free of the bias caused by the boundaries. Such truncation produces a $(d-2) \times(d-2)$ matrix and hence we only compute $d-2$ Lyapunov exponents. This, however, is of no consequence, since we rescale these exponents to estimate the spectrum density of the whole system as described above. If the coupling is over more than one site, one needs to discard an appropriate number of rows and columns [25].

In effect we can think of this procedure as using data from a window of size $d$ to obtain an accurate estimate of the Jacobian of a sub-system of size $d-2$ (or $d-2 q$ if the coupling has range $q$ ). This point of view shows why it reasonable to expect the Lyapunov exponents computed from the truncated Jacobians to give a reasonable estimate of the Lyapunov density of the whole system. Although this argument is largely heuristic, our numerical results below suggest that our procedure is very effective in removing boundary artefacts, and that it leads to dramatically improved estimates for the sub-system LS.

\section{Numerical results for the estimation of the LS from time-series}

In this section we present the numerical results when the LS is determined from a time-series. Let us 
first compare the results obtained in Section 2 for the known dynamics of the coupled logistic lattice with their reconstructed counterpart. The local dynamics for this example is the fully chaotic logistic map $f(x)=4 x(1-x)$ with coupling $\epsilon=0.45$ and lattice size $N=100$. The evolution for these parameters is known to be in the fully chaotic spatio-temporal regime, i.e. the temporal evolution at each local site is chaotic and spatial correlations decay exponentially. We sample 20 neighbouring sites after discarding transient behaviour and keep 11000 time steps. Our sample set is thus $20 \times 11000$ long. It is important to note that we are using the local state variables themselves as observables. In general the observables may be non-trivially dependent on the state variables.

Using spatial delay reconstructions $d=1, \ldots, 20$ we calculated sub-system LS using either linear fits (L-fit) or linear-quadratic fit (LQ-fit) as in Eq. (13) and in each case with and without discarding the outer layer of the Jacobians. In all these cases we used data from 10000 time steps to fit the dynamics, $d+20$ nearest neighbours in the L-fits and $2 d+20$ nearest neighbours in the LQ-fits. These parameters were chosen after considerable preliminary exploration and appeared to give robust and repeatable results. We used 1000 time steps to estimate the sub-system LS from the estimated Jacobians. Our experience with computing the spectrum when the dynamics is known [21] indicates that this is sufficient to ensure convergence. Of course, estimating Lyapunov exponents from data, and in particular negative exponents, is known to be a difficult task, and it is thus reasonable to ask how accurate and reliable our results are. We address this by comparing to computations carried out by assuming full knowledge of the dynamics, both in Fig. 1, and in [21].

Thus, in Fig. 2 we depict the interleaving properties for the different ways of calculating the Jacobians. The interleaving works better for the LQ-fit (Fig. 2c and d) than for the L-fit (Fig. 2a and b) and even better when using discarded outer layers in the Jacobians (Fig. 2d). This is especially clear for the most negative exponents.

In Fig. 3 we have plotted the corresponding convergence to the limit curve for the four possible methods using the rescaling in Eq. (10). The limit curve is computed using full knowledge of the dynamics, as in Fig. 1b. In all the cases the upper part of the spectrum is well approximated when $d$ is large enough. It is clear though, that using discarded Jacobians gives a much faster convergence for the upper part of the sub-system LS (compare Fig. 3b and d to Fig. 3a and c). Using the LQ-fit with discarded Jacobians leads to a very good approximation to the whole LS even for small $d$ (Fig. 3d).

Now we use these approximations of the limit curve to extract estimates of the largest Lyapunov exponent, Lyapunov dimension density and KS entropy density using both the direct method and the polygon method discussed earlier.

In Fig. 4 we have plotted estimates of the largest Lyapunov exponent using (a) the direct method and (b) the polygon method. We see that the largest Lyapunov exponent is well approximated using both approaches. Both L-fits and LQ-fits work well in these cases. The success of the direct method in low dimensions can be explained by considering the fits that we are actually performing when approximating the Jacobians. When $d$ is low we are able to find true nearest neighbours, and are indeed doing a local approximation of the dynamics, hence finding Jacobians in some reconstructed space. When $d$ is higher this is no longer possible. However, if the map can be approximated globally in the chosen basis, the Jacobians are still well approximated in high dimensions.

The fact that the largest Lyapunov exponent can be approximated well in low dimensions by local fits suggests that we can use these estimates as an upper bound for the estimates of the whole LS from the global fit.

Similarly we compare the efficiency of different approaches in the estimation of the Lyapunov dimension density as depicted in Fig. 5. We see that only when using the polygon method as in Fig. $5 \mathrm{~b}$ are we able to get a good estimate of $\rho_{d}$ and the estimate is improved further by discarding the edges of the Jacobians. The failure of the direct method to estimate $\rho_{d}$ comes from the second term in Eq. (3) which is used in Eq. (7). A small error in the denominator in Eq. (3) amplifies the error in the estimate of $\rho_{d}$. This implies that the poly- 

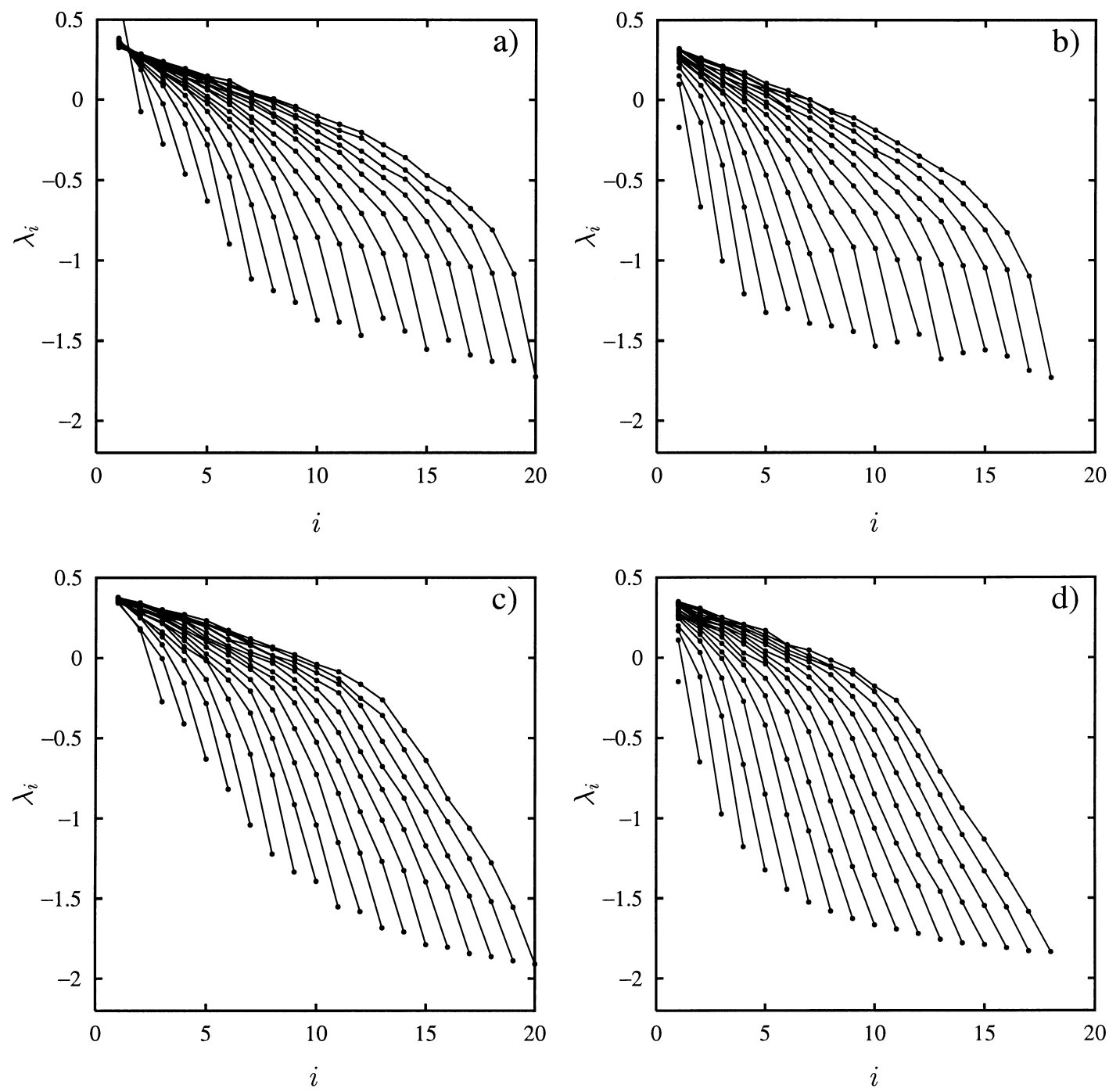

Fig. 2. Interleaving properties for sub-system sizes $d=1, \ldots, 20$ using spatial delay reconstructions and different methods of estimating the Jacobians for the same coupled map lattice as in Fig. 1. (a) L-fit without discarding the edges of the Jacobians. (b) LQ-fit without discarding. (c) L-fit with discarding. (d) LQ-fit with discarding.

gon method is preferable to the direct method when estimating $\rho_{d}$ from time-series.

In the case of KS entropy density (see Fig. 6), the polygon method works best, but in this case the direct method also gives good estimates. The discarded edges in the Jacobians improve the estimate for both methods.

The results suggest that when trying to approximate the whole LS we should consider the fitting as global, and therefore it becomes important to disregard the outer layer of the approximated Jacobian and use the polygon method.

Typically, the underlying system will not be contained in the chosen basis. To investigate this further we looked at time-series taken from two other spatially extended systems, where the local map is not contained in the fitting basis. First, we used Eq. (1) with the skewed logistic map as the local dynamics, 

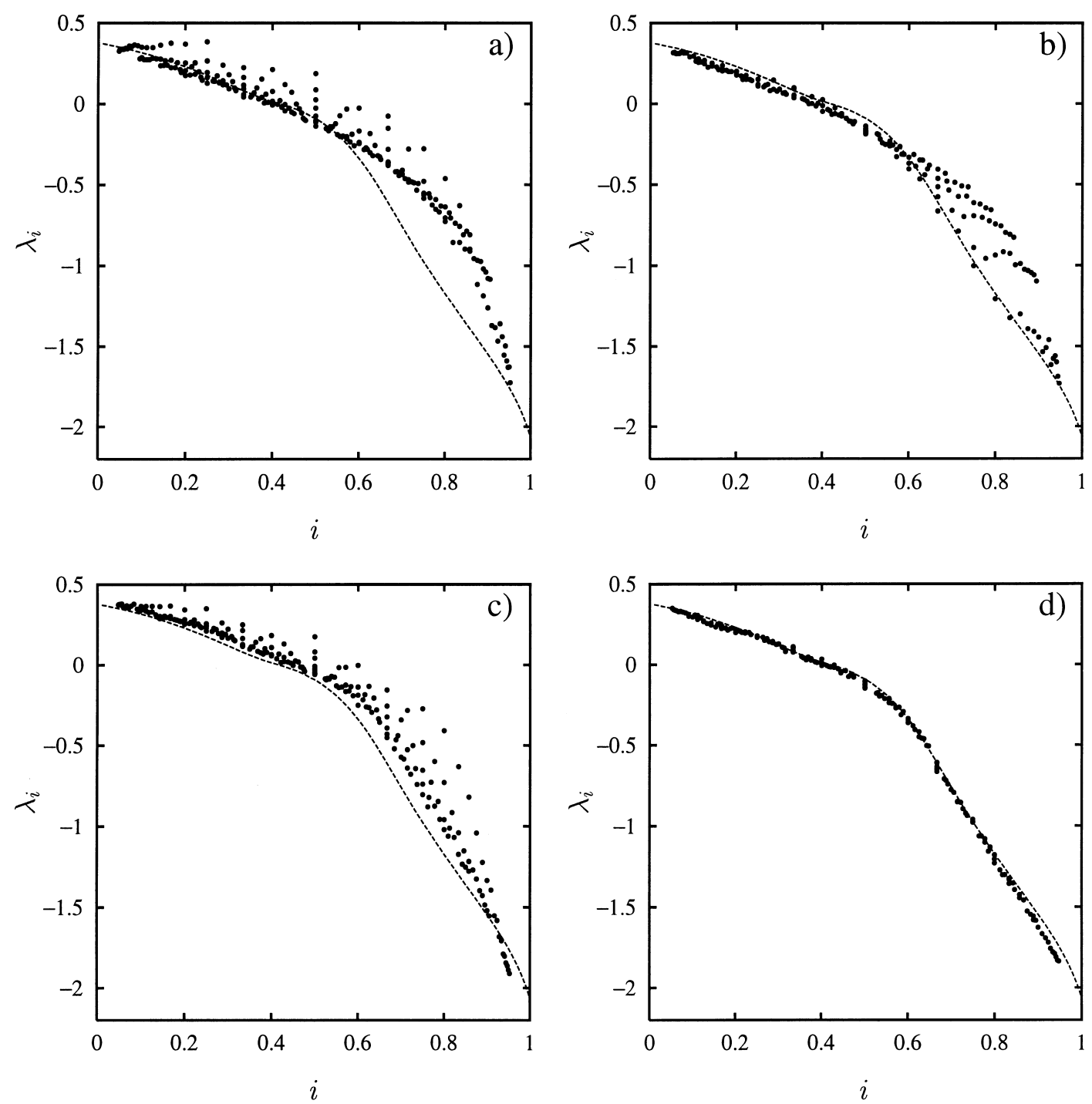

Fig. 3. Estimated sub-system LS for sub-system sizes $d=1, \ldots, 20$ using spatial delay reconstructions and different ways of estimating the Jacobians for the same coupled map lattice as in Fig. 1. (a) L-fit without discarding the edges of the Jacobians. (b) LQ-fit without discarding. (c) L-fit with discarding. (d) LQ-fit with discarding. The limit curve is computed the real dynamics, and hence is the same as in Fig. 1b.

i.e.

$f(x)=\frac{-1+a b(2 x-1)+\sqrt{(1+a b)^{2}-4 a b x}}{2 a b^{2}}$,

where $a$ is a nonlinearity parameter (it comes from the $a$ in $a x(1-x))$ and $b$ a skewness parameter. When $b=0$ we recover the familiar logistic map studied above and as $b$ is moved away from this value, the map is then skewed to one side. The parameter is bounded by $-1 / a<b<1 / a$ as in these limits the derivative becomes infinite at 0 and 1 . We study here the case $b=0.2$ corresponding to a high degree of skewness, still with $a=4$.

As a further example we also study the following local map

$f(x)=x \mathrm{e}^{r(1-x)}$, 

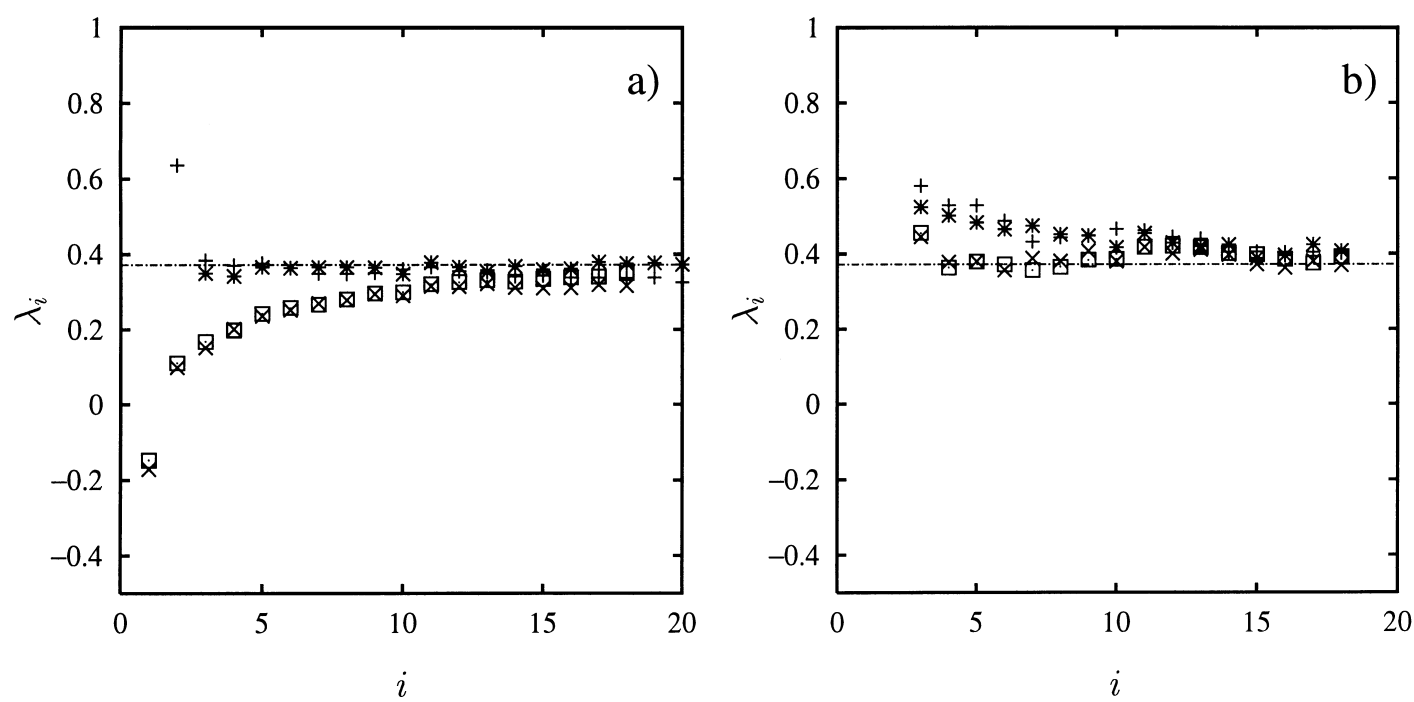

Fig. 4. Estimated largest Lyapunov exponent for the same coupled map lattice as in Fig. 1 using (a) the direct method; (b) the polygon method. Different methods of approximating the Jacobian are indicated by: $(+)$ L-fit without discarded edges, $(\times)$ L-fit with discarded edges, (*) LQ-fit without discarded edges, ( $\square$ ) LQ-fit with discarded edges.

using $r=4.5$. In both cases we used the coupling parameter $\epsilon=0.40$.

We sampled 20 neighbouring sites as before and repeated the calculations to obtain approximations

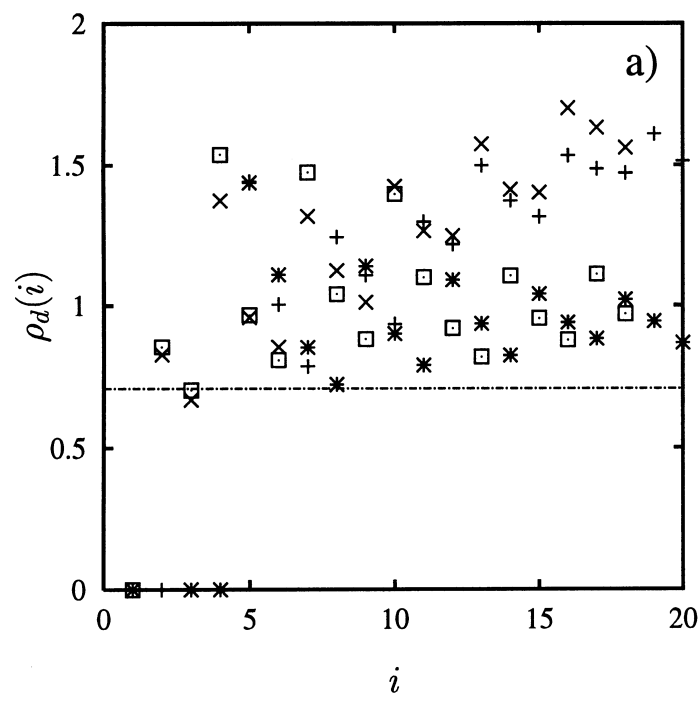

of sub-system Lyapunov spectra. From these we extracted estimates of $\lambda_{1}, \rho_{d}$ and $\rho_{h}$. We used the same choice of basis as before (L-fit and LQ-fit). As previously noted, using the polygon method and

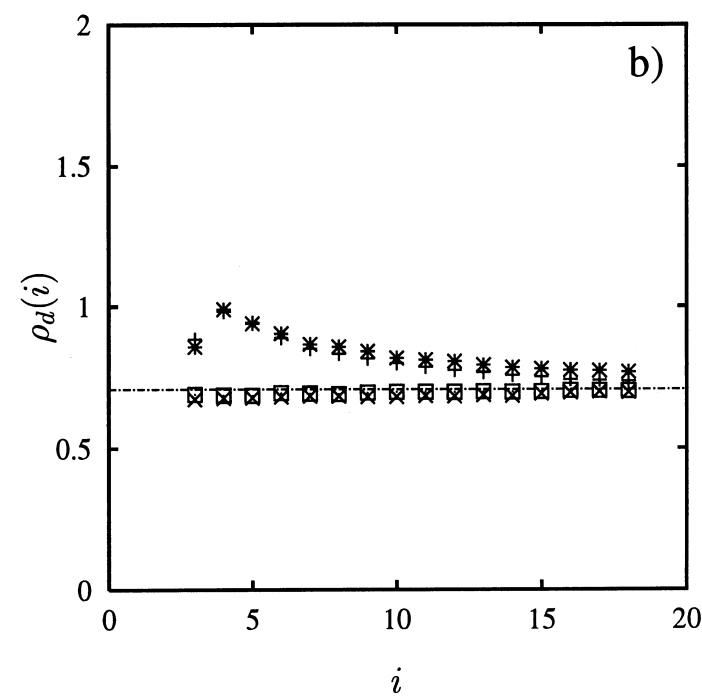

Fig. 5. Estimated Lyapunov dimension density for the same coupled map lattice as in Fig. 1 using (a) the direct method; (b) the polygon method. Different methods of approximating the Jacobian are indicated by: $(+)$ L-fit without discarded edges, $(\times)$ L-fit with discarded edges, (*) LQ-fit without discarded edges, ( $\square$ ) LQ-fit with discarded edges. 

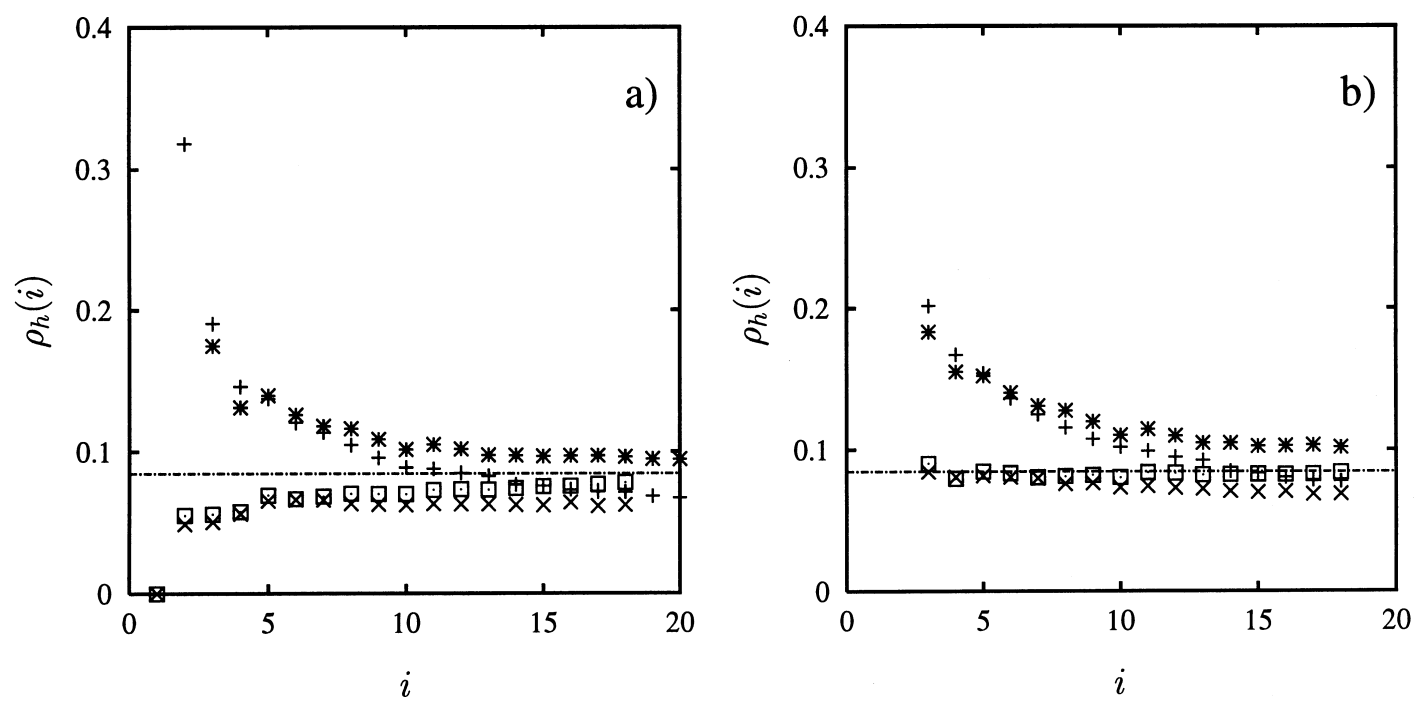

Fig. 6. Estimated KS entropy density for the same coupled map lattice as in Fig. 1 using (a) the direct method; (b) the polygon method. Different methods of approximating the Jacobian are indicated by: $(+)$ L-fit without discarded edges, $(\times)$ L-fit with discarded edges, $(*)$ LQ-fit without discarded edges, ( $\square$ ) LQ-fit with discarded edges.

Jacobians with discarded edges gave consistently better results; we thus restrict our next analysis to this approach.

In Fig. 7 we depict the interleaving and convergence to the limiting LS curve for the CML with

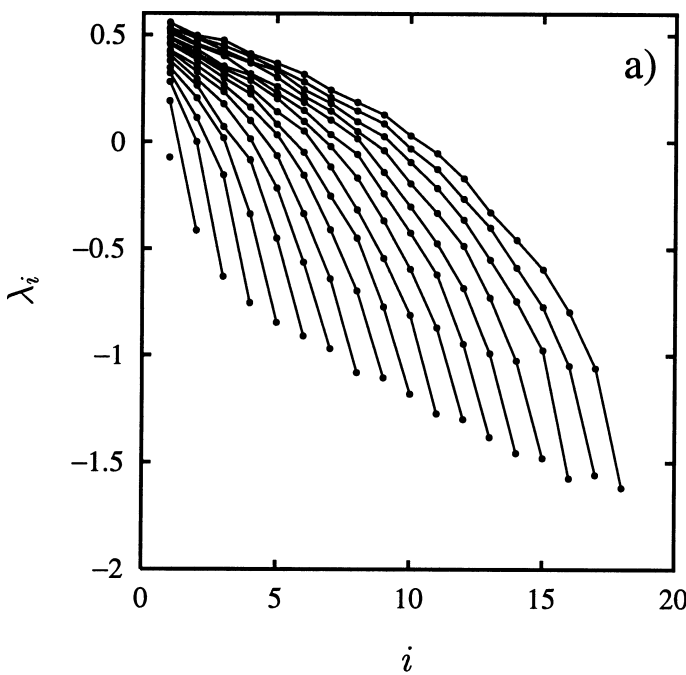

local skewed logistic maps. We present results for the LQ fit; those for the L-fit were somewhat worse and are not shown. As can be observed from a careful examination of Fig. 7a interleaving in this case is not fully satisfied. Moreover, from Fig. $7 \mathrm{~b}$ we note that

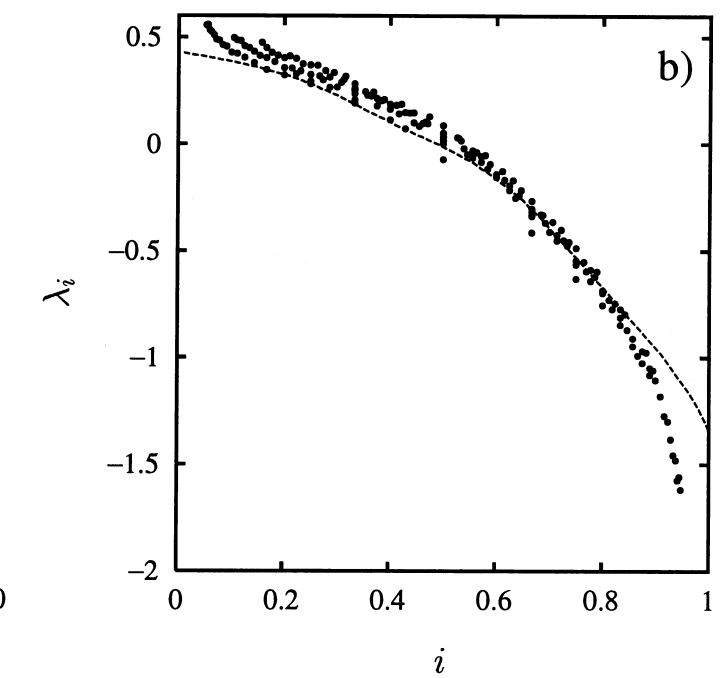

Fig. 7. Estimated sub-system LS from time-series for increasing space delay reconstructions for the coupled map lattice with $\epsilon=0.4$ using the skewed logistic map (14) with $a=4, b=0.2$ as local dynamics. (a) Interleaving when using LQ-fit. (b) Convergence for LQ-fit. In (b) the exact LS for the whole system, calculated assuming knowledge of the true dynamics, is super-imposed as a continuous curve. 

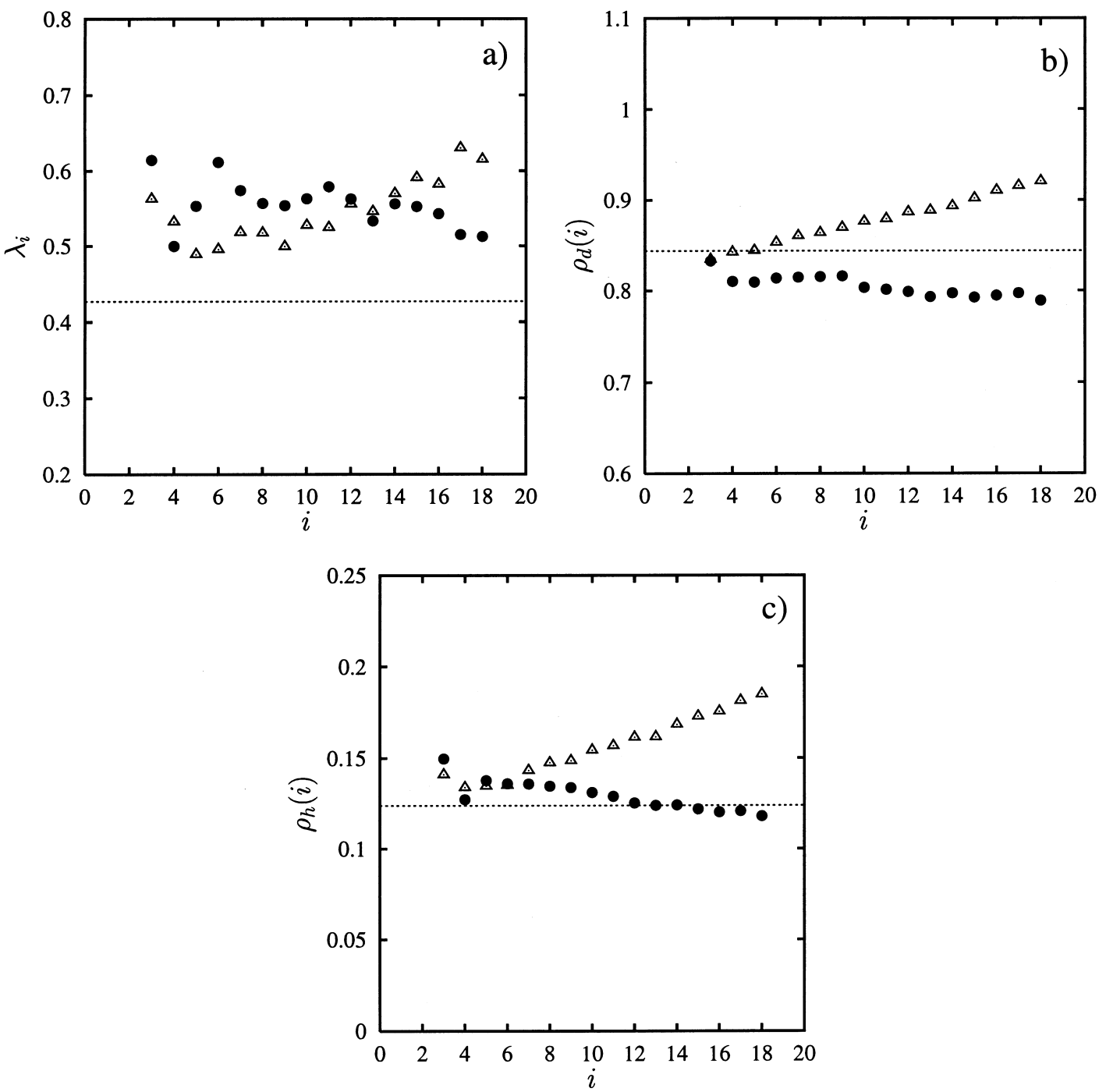

Fig. 8. Estimation for the same system as in Fig. 7 of (a) largest Lyapunov exponent; (b) Lyapunov dimension density; (c) KS entropy density for the skewed system. Different fits are indicated by: $(\triangle)$ L-fit with discarded edges, $(\mathbf{O})$ LQ-fit with discarded edges.

the convergence towards the real limit curve of the LS fails, especially for the most negative Lyapunov exponents.

The estimates for $\lambda_{1}, \rho_{d}$ and $\rho_{h}$ for the skewed logistic lattice are given in Fig. 8. The estimate for $\lambda_{1}$ is a bit higher than the real value $(0.427)$ and the LQ-fit does not perform significantly better than the L-fit (Fig. 8b). The estimates for $\rho_{d}$ and $\rho_{h}$ are also worse than when using the (un-skewed) logistic map as the underlying local dynamics. However, the real value of $\rho_{d}(0.844)$ is not too badly approximated (Fig. 8b). In general, we see that as the underlying dynamics moves away from the space generated by the basis used to fit the dynamics (L-fit or LQ-fit), the results deteriorate. In this particular case, the results are still acceptable since the skewed logistic map can be reproduced reasonably well by the LQ basis. 

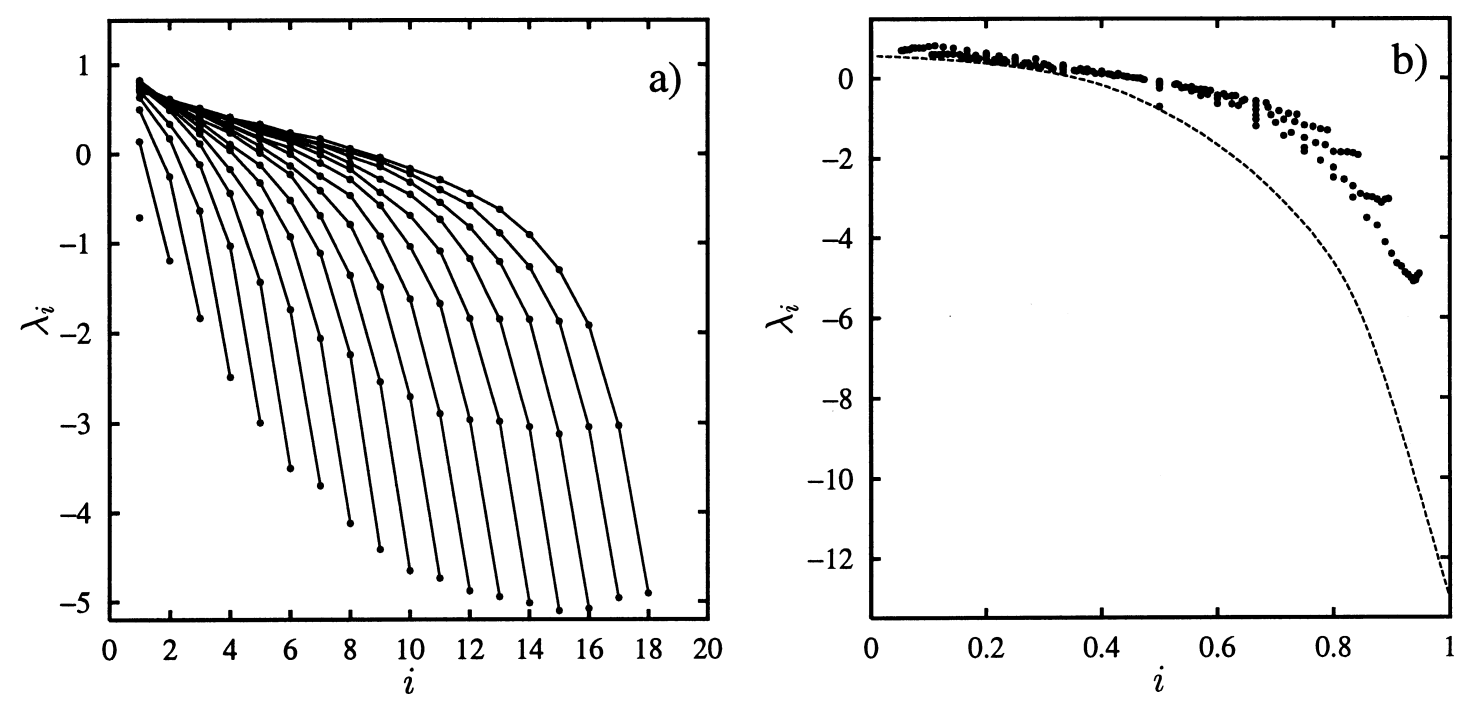

Fig. 9. Interleaving and estimated sub-system LS from time-series for increasing space delay reconstructions for the coupled map lattice with $\epsilon=0.4$ using the exponential map (15) with $r=4.5$ as local dynamics. (a) Interleaving when using L-fit. (b) Convergence for L-fit. In (b) the exact LS for the whole system, calculated assuming knowledge of the true dynamics, is super-imposed as a continuous curve.

To enhance the effects of the choice of the underlying system, we now consider the exponential map as the local dynamics. We continue using the L-fit and LQ-fit to estimate Jacobians (discarding the outer layer as before). In this case it is clear that the basis is far from being a reasonable representation for the dynamics.

Again we calculated the sub-system LS for increasing spatial delay reconstructions. The resulting interleaving and convergence is shown in Fig. 9. The interleaving (Fig. 9a) is still good for small $d$ but starts to fail as $d \approx 10$ or greater. This reflects the fact that in small dimensions, the approximation is local (so it is good). For large $d$ the approximation is global and obviously bad for the exponential map. This is reflected in the convergence plot (Fig. 9b). There is no convergence for the negative part of the LS. In this case the L-fit performs much better than the LQ-fit (not shown) and seems to converge to the whole LS in the upper part.

As before, we use the convergence plots with the polygon method to estimate $\lambda_{1}, \rho_{d}$ and $\rho_{h}$ as shown in Fig. 10. Here we clearly see that in all the three cases the LQ-fit does not give any sensible results.

\section{Discussion}

We have developed an algorithm to estimate intensive measures such as the largest Lyapunov exponent, Lyapunov dimension density and KS entropy density in spatially extended systems from time-series sampled from sufficiently large sub-systems. Our approach is based on the rescaling and interleaving of the sub-system Lyapunov spectra and a careful consideration of errors introduced by boundary effects.

The performance of the algorithm is governed by several factors. Firstly, the estimation of the Jacobians has to be performed using a global fit, and care must go into choosing the right basis. Time-delay plots might be one way of obtaining an idea of a suitable basis. Further work in this direction is in progress [32]. Secondly, we find that discarding the outer layer of the Jacobian at each time step allows us to eliminate what would otherwise be noisy input, and significantly improves the quality of the resulting spectrum. Thirdly, care needs to be taken in extracting the intensive quantities from sub-system spectra. We have presented an optimal rescaling for the sub-system spectrum and suggested an accompanying polygon 

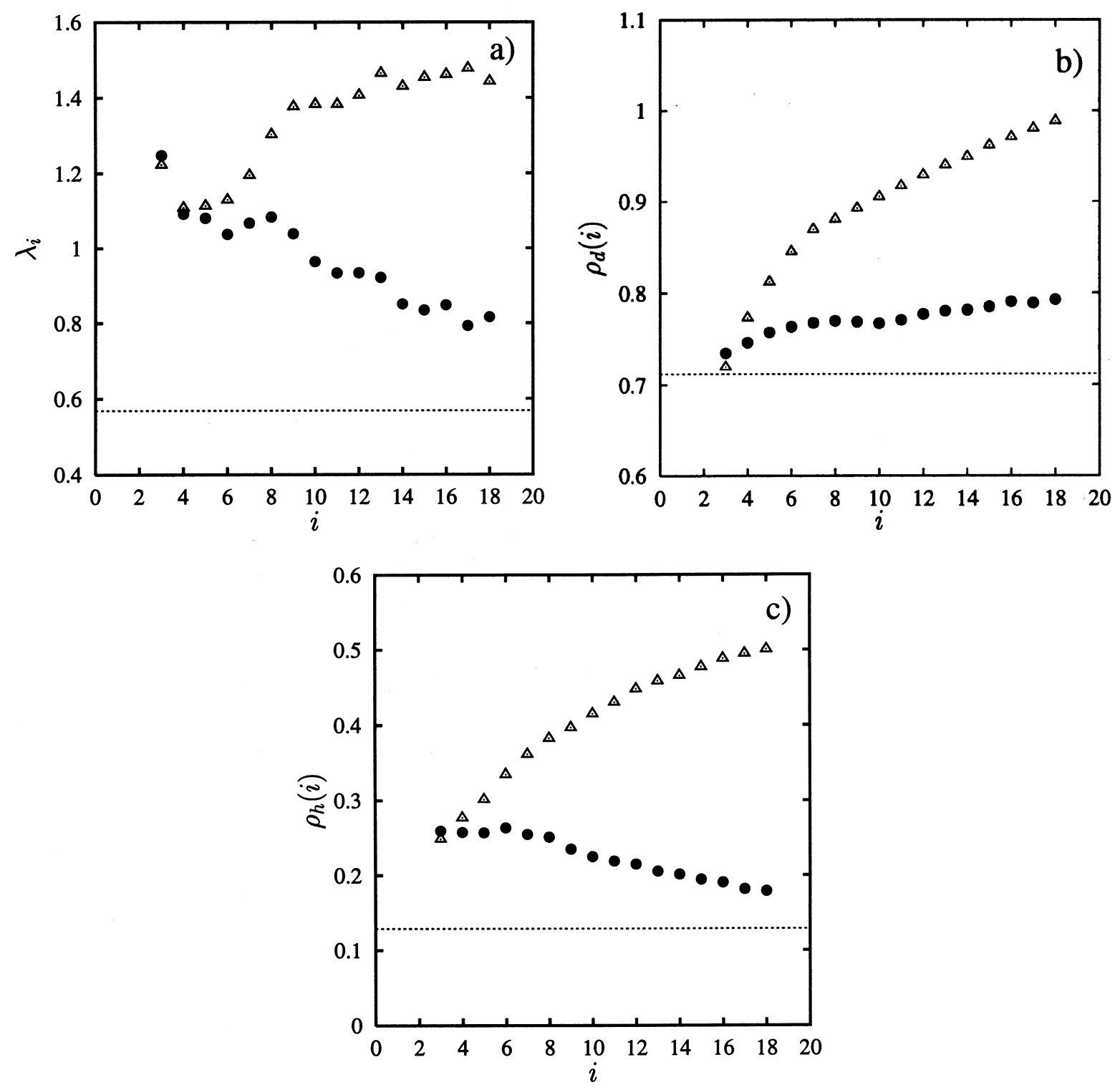

Fig. 10. Estimation for the same system as in Fig. 9 of (a) largest Lyapunov exponent; (b) Lyapunov dimension density; (c) KS entropy density for the exponential system. Different fits are indicated by: $(\triangle)$ L-fit with discarded edges, $(\mathbf{O})$ LQ-fit with discarded edges.

interpolation scheme for calculating the Lyapunov dimension and KS entropy densities.

For choices of local dynamics that are not well approximated in the chosen basis, we find that estimates of the Lyapunov dimension density and KS entropy density are poor. A natural way of addressing this is to use the near-diagonal structure of the Jacobian to per- form local estimates in much lower dimensions. Thus, in particular, for the nearest neighbour coupling used here, the Jacobian is tri-diagonal, and we only need estimate three terms in each row, using an embedding dimension of three. Furthermore, since the system is spatially homogenous, one can ignore the spatial position of a sample data point when looking for nearest 
neighbours, thereby dramatically increasing the effective size of the available data. This allows us to use genuinely local estimates in low embedding dimensions, and negates the need to find a basis adapted to the dynamics [25]. This is also the technique adopted by Bünner and Hegger [23], and as already indicated we are currently incorporating this idea into the approach presented here [25].

\section{Acknowledgements}

This work was in part carried out under a UK Engineering and Physical Sciences Research Council grant number GR/L42513. The authors would like to thank D. Broomhead, J. Huke and T. Schreiber for stimulating discussions and helpful suggestions.

\section{References}

[1] M.R. Muldoon, D.S. Broomhead, J.P. Huke, Delay reconstruction for multiprobe signals, IEE Digest 143 (1994) $3 / 1-3 / 5$.

[2] D. Pritchard, J. Theiler, Generating surrogate data for time series with several simultaneously measured variables, Phys. Rev. Lett. 73 (1994) 951-954.

[3] D.A. Rand, H.B. Wilson, Using spatio-temporal chaos and intermediate-scale determinism to quantify spatially extended ecosystems, Proc. R. Soc. London B 259 (1995) 111-117.

[4] S.A.R.B. Rombouts, R.W.M. Keunen, C.J. Stam, Investigation of nonlinear structure in multichannel EEG, Phys. Lett. A 202 (1995) 352-358.

[5] S. Little, S. Ellner, M. Pascual, M. Neubert, D. Kaplan, T. Sauer, H. Caswell, A. Solow, Detecting nonlinear dynamics in spatio-temporal systems, examples from ecological models, Physica D 96 (1996) 321-333.

[6] S.J. Schiff, P. So, T. Chang, R.E. Burke, T. Sauer, Detecting dynamical interdependence and generalized synchrony through mutual prediction in a neural ensemble, Phys. Rev. E 54 (1996) 6708-6724.

[7] S. Ørstavik, J. Stark, Reconstruction and cross-prediction in coupled map lattices using spatio-temporal embedding techniques, Phys. Lett. A 247 (1998) 146-160.

[8] H. Kantz, T. Schreiber, Nonlinear Time Series Analysis, 2nd Edition, Cambridge University Press, Cambridge, 1999.

[9] P. Grassberger, I. Procaccia, Characterization of strange attractors, Phys. Rev. Lett. 50 (1983) 346-349.

[10] J.-P. Eckmann, D. Ruelle, Ergodic theory of chaos and strange attractors, Rev. Mod. Phys. 57 (1985) 617-656.

[11] H. Kantz, E. Olbrich, Scalar observations from a class of high-dimensional chaotic systems: limitations of the time delay embedding, Chaos 7 (1997) 423-429.
[12] P. Grassberger, Information content and predictability of lumped and distributed dynamical systems, Phys. Scripta 40 (1989) 346-353.

[13] G. Mayer-Kress, K. Kaneko, Spatiotemporal chaos and noise, J. Statist. Phys. 54 (1989) 1489-1508.

[14] M. Bauer, H. Heng, W. Martienssen, Characterisation of spatiotemporal chaos from time series, Phys. Rev. Lett. 71 (1993) 521-524.

[15] L.S. Tsimring, Nested strange attractors in spatiotemporal chaotic systems, Phys. Rev. E 48 (1993) 3421-3426.

[16] A. Torcini, A. Politi, G.P. Puccioni, G. D'Alessandro, Fractal dimension of spatially extended systems, Physica D 53 (1991) 85-101.

[17] R. Carretero-González, S. Ørstavik, J. Huke, D.S. Broomhead, J. Stark, Thermodynamic limit from small lattices of coupled maps, Phys. Rev. Lett. 83 (1999) 3633-3636.

[18] K. Kaneko, Towards thermodynamics of spatiotemporal chaos, Prog. Theoret. Phys. Suppl. 99 (1989) 263-287.

[19] D. Ruelle, Large volume limit distribution of characteristic exponents in turbulence, Commun. Math. Phys. 87 (1982) 287-302.

[20] N. Parekh, V.R. Kumar, B.D. Kulkarni, Synchronization and control of spatiotemporal chaos using time-series data from local regions, Chaos 8 (1998) 300-306.

[21] R. Carretero-González, S. Ørstavik, J. Huke, D.S. Broomhead, J. Stark, Scaling and interleaving of sub-system Lyapunov exponents for spatio-temporal systems, Chaos 9 (1999) 466486.

[22] B.R. Parlett, The Symmetric Eigenvalue Problem, PrenticeHall, Englewood Cliffs, NJ, 1980.

[23] M.J. Bünner, R. Hegger, Estimation of Lyapunov spectra from space-time data, Phys. Lett. A 258 (1) (1999) 25-30.

[24] H. Voss, M.J. Bünner, M. Abel, Identification of continuous, spatiotemporal systems, Phys. Rev. E 57 (1) (1998) 28202823.

[25] R. Carretero-González, S. Ørstavik, J. Stark, A quasi-diagonal approach to the estimation of Lyapunov spectra for spatio-temporal systems from multivariate time series, Phys. Rev. E, to appear.

[26] K. Kaneko, Theory and Applications of Coupled Map Lattices, Wiley, New York, 1993.

[27] V.I. Oseledec, A multiplicative ergodic theorem. Ljapunov characteristic numbers for dynamical systems, Trans. Moscow Math. Soc. 19 (1968) 197-231.

[28] J.L. Kaplan, J.A. Yorke, Chaotic behaviour of multidimensional difference equations, Lect. Notes Math. 730 (1979) 204-227.

[29] P. Manneville, Liapunov exponents for the KuramotoSivashinsky model, Lect. Notes Phys. 230 (1985) 319326.

[30] F. Takens, Detecting strange attractors in turbulence, Lect. Notes Math. 989 (1981) 366-381.

[31] H.F. von Bremen, F.E. Udwadia, W. Proskurowski, An efficient QR based method for the computation of Lyapunov exponents, Physica D 101 (1997) 1-16.

[32] R. Carretero-González, Extracting local dynamics from extended dynamical systems: a times-series reconstruction perspective, in preparation. 